J. Korean Math. Soc. 52 (2015), No. 3, pp. 489-501

http://dx.doi.org/10.4134/JKMS.2015.52.3.489

\title{
DUO RING PROPERTY RESTRICTED TO GROUPS OF UNITS
}

\author{
Juncheol Han, Yang Lee, and Sangwon Park
}

\begin{abstract}
We study the structure of right duo ring property when it is restricted within the group of units, and introduce the concept of right unit-duo. This newly introduced property is first observed to be not left-right symmetric, and we examine several conditions to ensure the symmetry. Right unit-duo rings are next proved to be Abelian, by help of which the class of noncommutative right unit-duo rings of minimal order is completely determined up to isomorphism. We also investigate some properties of right unit-duo rings which are concerned with annihilating conditions.
\end{abstract}

Throughout this paper all rings are associative with identity unless otherwise stated. Let $R$ be a ring. $X(R)$ denotes the set of all nonzero nonunits in $R$, and $G(R)$ denotes the group of all units in $R$. For $a \in R$, set $[a]_{\ell}=\{u a \mid u \in G(R)\}$ and $[a]_{r}=\{a u \mid u \in G(R)\}$, i.e., $[a]_{\ell}=G(R) a$ and $[a]_{r}=a G(R)$. Let $J(R)$, $I(R)$, and $N(R)$ denote the Jacobson radical, the set of all idempotents, and the set of all nilpotent elements in $R$, respectively. $|S|$ denotes the cardinality of a subset $S$ of $R$. Write $R^{*}=R \backslash\{0\}$. $\mathbb{Z}\left(\mathbb{Z}_{n}\right)$ denotes the ring of integers $\left(\right.$ modulo $n$ ). $\mathbb{Q}$ denotes the field of rational numbers. $G F\left(p^{n}\right)$ denotes the Galois field of order $p^{n}$.

Denote the $n$ by $n$ full (resp., upper triangular) matrix ring over $R$ by $\operatorname{Mat}_{n}(R)$ (resp., $U_{n}(R)$ ) and use $e_{i j}$ for the matrix with $(i, j)$-entry 1 and elsewhere 0. Following the literature, we write $D_{n}(R)=\left\{\left(a_{i j}\right) \in U_{n}(R) \mid\right.$ all diagonal entries are equal $\}$ and $V_{n}(R)=\left\{\left(a_{i j}\right) \in D_{n}(R) \mid a_{1 k}=a_{2(k+1)}=\right.$ $\cdots=a_{h n}$ for $h=1,2, \ldots, n-1$ and $\left.k=2, \ldots, n\right\}$. Note $V_{n}(R) \cong \frac{R[x]}{x^{n} R[x]}$, so $V_{n}(R)$ is commutative if so is $R$.

Due to Feller [7], a ring is called right (resp. left) duo if every right (resp. left) ideal is an ideal; a ring is called $d u o$ if it is both right and left duo. It is easily shown that idempotents of right duo rings are central. There are very useful results for duo rings in $[3,16,24]$.

Received July 10, 2014; Revised December 24, 2014.

2010 Mathematics Subject Classification. 16U80, 16D25, 16U60, 16W22.

Key words and phrases. right unit-duo ring, right duo ring, right unit-duo ring of minimal order, left-right symmetric, annihilator, group ring. 
We next consider right duo property on the group of units in rings. A ring $R$ will be called right unit-duo if $[a]_{\ell} \subseteq[a]_{r}$ for every $a \in R$. Left unit-duo rings are defined similarly. A ring will be called unit-duo if it is both left and right unit-duo. Note that a ring $R$ is unit-duo if and only if $[a]_{\ell}=[a]_{r}$ for each $a \in R$. It is easily checked that both commutative rings and finite direct products of division rings are unit-duo. It is also obvious that a ring $R$ is unit-duo when $G(R)$ is contained in the center of $R$. Note that a ring $R$, with $X(R)$ nonempty, is right unit-duo if and only if $[a]_{\ell} \subseteq[a]_{r}$ for every $a \in X(R)$. Indeed, $[u]_{\ell}=[u]_{r}=G(R)$ for every $u \in G(R)$, and $[0]_{\ell}=[0]_{r}=\{0\}$. When $R$ is a unit-duo ring, we use $[a]$ to denote $[a]_{\ell}=[a]_{r}$ for every $a \in R$. In the following section we see that this newly introduced ring property is not left-right symmetric.

\section{Basic structure of right unit-duo rings}

In this section, we observe basic properties and examples of right unit-duo rings. We also see the relationships among right duo, right unit-duo, and Abelian rings. In the procedure we examine the unit-duo property of some kind of group ring.

We first observe that the unit-duo property is not left-right symmetric. To do it, we apply the construction of ring in [22, Definition 1.3] and argument in [15, pages 6,7$]$. Let $R$ be a commutative ring with an endomorphism $\sigma$ and $M$ be an $R$-module. For $R \oplus M$, the addition and multiplication are given by

$$
\begin{aligned}
& \left(r_{1}, m_{1}\right)+\left(r_{2}, m_{2}\right)=\left(r_{1}+r_{2}, m_{1}+m_{2}\right) \text { and } \\
& \left(r_{1}, m_{1}\right)\left(r_{2}, m_{2}\right)=\left(r_{1} r_{2}, \sigma\left(r_{1}\right) m_{2}+m_{1} r_{2}\right) .
\end{aligned}
$$

Then this construction forms a ring and usually called the skew-trivial extension of $R$ by $M$, denoted by $R \propto M$.

Theorem 1.1. (1) Let $K$ be a field with a monomorphism $\sigma$ and $M$ be a $K$ module. If $\sigma$ is not surjective, then $K \propto M$ is a right unit-duo ring which is not left unit-duo.

(2) Let $K$ be a field with a monomorphism $\sigma$ and $M$ be a $K$-module. If $\sigma$ is bijective, then $K \propto M$ is a unit-duo ring.

Proof. (1) Let $R=K \propto M$ and assume that $\sigma$ is not surjective. Since $J(R)=$ $0 \oplus M$ and $R / J(R) \cong K, G(R)=\{(r, m) \in R \mid r \neq 0\}$. By assumption, there exists some $s \in K$ such that $s \notin \sigma(K)$. Then $(s, n) \in G(R)$ for any $n \in M$. Consider $0 \neq(0, m) \in R$. Then

$$
(0, m)(s, n)=(0, m s)=(0, s m) \neq(0, \sigma(t) m)=(t, n)(0, m)
$$

for any $(t, n) \in R$ since $s \notin \sigma(K)$. This means $(0, m)(s, n) \notin G(R)(0, m)$, and hence $R$ is not a left unit-duo ring.

To show that $R$ is right unit-duo, let $(s, m) \in G(R),(0, n) \in X(R)$. Then

$$
(s, m)(0, n)=(0, \sigma(s) n)=(0, n \sigma(s))=(0, n)(\sigma(s), 0) .
$$


Here $(\sigma(s), 0) \in G(R)$ since $s \neq 0$, and so $R$ is right unit-duo.

(2) The proof is similar to (1).

In the following we see an illustration of Theorem 1.1.

Example 1.2. (1) Let $K$ be a field and $K(x)$ be the quotient field of $K[x]$. Recall the non-surjective monomorphism of $K(x)$ defined by $\sigma\left(\frac{f(x)}{g(x)}\right)=\frac{f\left(x^{2}\right)}{g\left(x^{2}\right)}$. Set $R=K \propto K(x)$. Then $R$ is right but not left unit-duo. Note that $R$ is isomorphic to the subring

$$
R=\left\{\left(\begin{array}{cc}
h(x) & 0 \\
k(x) & \sigma((h(x))
\end{array}\right) \mid h(x), k(x) \in K(x)\right\}
$$

of $\operatorname{Mat}_{2}(K(x))$.

(2) For $R \oplus M$, the addition and multiplication are given by $\left(r_{1}, m_{1}\right)+$ $\left(r_{2}, m_{2}\right)=\left(r_{1}+r_{2}, m_{1}+m_{2}\right)$ and suppose that

$$
\left(r_{1}, m_{1}\right)\left(r_{2}, m_{2}\right)=\left(r_{1} r_{2}, r_{1} m_{2}+m_{1} \sigma\left(r_{2}\right)\right) .
$$

Then this construction also forms a ring. Let $R_{r}=K \oplus K(x)$ with this multiplication, where $K$ and $K(x)$ are such as in (1). We can show that $R_{r}$ is not right but left unit-duo via a similar computation to (1). Note that $R_{r}$ is isomorphic to the subring

$$
R=\left\{\left(\begin{array}{cc}
h(x) & k(x) \\
0 & \sigma((h(x))
\end{array}\right) \mid h(x), k(x) \in K(x)\right\}
$$

of $\operatorname{Mat}_{2}(K(x))$.

A ring is usually called Abelian if each idempotent is central. A ring is usually called reduced if it has no nonzero nilpotent elements. Reduced rings are easily shown to be Abelian. Recall that an involution on a ring $R$ is a function $*: R \rightarrow R$ which satisfies the properties that $(x+y)^{*}=x^{*}+y^{*}$, $(x y)^{*}=y^{*} x^{*}, 1^{*}=1$, and $\left(x^{*}\right)^{*}=x$ for all $x, y \in R$.

Theorem 1.3. (1) Right (left) unit-duo rings are Abelian.

(2) Every right Artinian, right (left) unit-duo simple ring is a division ring.

(3) Let $R$ be a domain. If $R$ is right duo, then $R$ is right unit-duo.

(4) Let $R$ be a ring with an involution $*$. Then $R$ is left unit-duo if and only if it is right unit-duo.

Proof. (1) Let $R$ be a right unit-duo ring and $e^{2}=e \in R$. We first claim that if $f^{2}=f \in[e]_{r}$, then $e=f$. For, $f=e a$ for some $a \in G(R)$, entailing $f=e f$. From $f=e a$, we get $e a(1-f)=f(1-f)=0$. Since $R$ is right unit-duo, there exists $a^{\prime} \in G(R)$ such that $0=e a(1-f)=e(1-f) a^{\prime}$. This yields $e(1-f)=0$, so $e=e f$. Consequently, $e=f$.

Next consider an idempotent $u e u^{-1} \in R$ for $u \in G(R)$. Since $R$ is right unit-duo, ueu $u^{-1}=e u^{\prime} u^{-1} \in[e]_{r}$ for some $u^{\prime} \in G(R)$. Then we get $u e u^{-1}=e$ by the preceding claim, entailing $u e=e u$. 
We accordingly have that $b e=e b$ for any $b \in N(R)$ (hence $1-b \in G(R)$ ), via the computation $(1-b) e=e(1-b)$.

Now let $r \in R$ be arbitrary. Consider $x=\operatorname{er}(1-e), y=(1-e) r e \in N(R)$. Then $x^{2}=y^{2}=0$, and this yields $x=e x=x e=0$ and $y=y e=e y=0$ by help of the preceding result. Consequently we have $e r=e r e=r e$, and so $R$ is Abelian. The proof of the left case is similar. (2) is an immediate consequence of (1).

(3) Let $R$ be right duo and $0 \neq a \in R, u \in G(R)$. Then $v \in R$ such that $u a=a v$. Observe as well that $a=u^{-1} a v=a v^{\prime} v$ for some $v^{\prime} \in R$ also since $R$ is right duo. This implies $a\left(1-v v^{\prime}\right)=0$, and since $R$ is a domain we have $v v^{\prime}=1$, entailing $v \in G(R)$.

(4) Let $R$ be left unit-duo. For $a \in R$ and $u \in G(R)$. Observe first $(u a)^{*}=$ $a^{*} u^{*}$, noting $u^{*} \in G(R)$. Since $R$ is left unit-duo, $a^{*} u^{*}=v a^{*}$ for some $v \in$ $G(R)$. This yields

$$
u a=\left((u a)^{*}\right)^{*}=\left(a^{*} u^{*}\right)^{*}=\left(v a^{*}\right)^{*}=a v^{*},
$$

noting $v^{*} \in G(R)$. This leads us to conclude that $R$ is right unit-duo. The proof of the converse is analogous.

Note. There exists another proof of Theorem 1.3(1) which is done by using a direct computation as follows. Since $(1-e) r e\left(e^{2}=e, r \in R\right)$ is nilpotent, we have $g=1+(1-e) r e \in G(R)$ and $e g=e$. Since $R$ is right unit-duo, there exists $h \in G(R)$ such that $e=g e h$ (consider $\left.g^{-1} e \in[e]_{\ell}\right)$. Hence $e h=e e h=e g e h=$ $e e=e$, so from $e=g e h$ we obtain $e=g e$. Notice $g e=e+(1-e) r e$. This forces $(1-e) r e=g e-e=0$, which shows $r e=e r e$. Similarly we can obtain $e r=e r e$ from the nilpotent element $\operatorname{er}(1-e)$, proving that every idempotent is central.

Let $K$ be a commutative ring and $G$ be any group. Consider the standard involution $*$ on the group $\operatorname{ring} K G$ in [2], i.e., $\left(\sum a_{i} g_{i}\right)^{*}=\sum a_{i} g_{i}^{-1}$ for all $a_{i} \in K$ and $g_{i} \in G$. Then $K G$ is left unit-duo if and only if it is right unit-duo by Theorem 1.3(4). Let $Q_{8}$ be the quaternion group of order 8 , and consider the group ring $\mathbb{Z} Q_{8}$. Take $i+2 j \in \mathbb{Z} Q_{8}$ and we claim that $(i+2 j) i$ cannot be contained in $R(i+2 j)$, noting $i \in G\left(\mathbb{Z} Q_{8}\right)$. We see that every element of $\mathbb{Z} Q_{8}$ is of the form $\alpha=\sum_{s=0}^{3} a_{s} i^{s}+\left(\sum_{t=4}^{7} a_{t} i^{7-t}\right) j$, by using $Q_{8}=\langle i, j\rangle$. Letting $(i+2 j) i=\alpha(i+2 j)$, we get $a_{6}=-\frac{6}{15} \notin \mathbb{Z}$ by help of the computation in the proof of $\left[2\right.$, Example 1.2]. Thus $\mathbb{Z} Q_{8}$ is neither left nor right unit-duo by Theorem 1.3(4). But we will see that $\mathbb{Q} Q_{8}$ is unit-duo by Proposition 1.8 to follow.

The converse of Theorem 1.3(1) need not hold by the rings $R_{4}$ and $R_{5}$ of Example 2.2 to follow. The converse of Theorem 1.3(3) also need not hold by the ring of Example 1.7 to follow. In the following we find some conditions under which the converses holds.

Following [8], a ring $R$ is called von Neumann regular (simply, regular) (resp., unit-regular) if for every $x \in R$ there exists $y \in R$ (resp. $u \in G(R)$ ) such that 
$x y x=x($ resp. $x u x=x)$. Abelian regulars are unit-regular by [8, Corollary 4.2]. Let $R$ be a unit-regular ring and $a \in R$. Then $a=a u a$ for some $u \in$ $G(R)$. So if $R$ is Abelian, then $a=a u u^{-1} u a=($ auua $) u^{-1}=u^{-1}$ (auua) with auua $\in I(R)$. We use this fact freely. Due to Bell [1], a ring $R$ is called to satisfy the insertion-of-factors-property (simply, an IFP ring) if $a b=0$ implies $a R b=0$ for $a, b \in R$. Narbonne [19], Shin [23], and Xu-Xue [25] used the terms semicommutative, SI, and zero-insertive for the IFP, respectively. A ring is usually called reduced if it has no nonzero nilpotent elements. The class of IFP rings clearly contains commutative rings are reduced rings. It is shown that $D_{3}(R)$ is IFP if and only if $R$ is a reduced ring, by [12, Proposition 1.2] and [11, Proposition 2.8]. A simple computation yields that IFP rings are Abelian. It is easily checked that left or right duo rings are IFP.

Corollary 1.4. Let $R$ be a regular ring. Then the following conditions are equivalent:

(1) $R$ is right (left) unit-duo;

(2) $R$ is Abelian;

(3) $R$ is right (left) duo;

(4) $R$ is IFP.

Proof. Left or right unit-duo rings are Abelian by Theorem 1.3. For the proof of $(2) \Rightarrow(1)$, let $R$ be an Abelian regular ring and $x \in X(R)$. Then by [8, Corollary 4.2], $R$ is unit-regular, and so $x=u e=e u$ for some $u \in G(R)$ and $e \in I(R)$. We observe $[x]_{\ell}=[e]_{\ell}=[e]_{r}=[x]_{r}$ as well. Thus $R$ is unit-duo. $(2) \Rightarrow(3)$ is proved by $[8$, Theorem 3.2$]$. (4) $\Rightarrow(2)$ and $(3) \Rightarrow(4)$ are obvious.

By Corollary 1.4 and [8, Theorem 3.2], we also have that a regular ring is right unit-duo if and only if it is reduced.

Following Nicholson [21], a ring is called clean if every element is a clean element (i.e., a sum of a unit and an idempotent). The class of clean rings includes semiperfect rings and unit-regular rings $[4,5]$.

Proposition 1.5. Let $R$ be a clean ring such that $G(R)$ is an Abelian group. Then the following conditions are equivalent:

(1) $R$ is right (left) unit-duo;

(2) $R$ is a commutative ring;

(3) $R$ is right (left) duo;

(4) $R$ is Abelian.

Proof. (4) $\Rightarrow(2)$. Let $R$ be an Abelian ring and $x, y \in R$. Since $R$ is clean, $x=u_{x}+e_{x}, y=u_{y}+e_{y}$ for some $u_{x}, u_{y} \in G(R)$ and $e_{x}, e_{y} \in I(R)$. Since $R$ is Abelian, $e_{x} e_{y}=e_{y} e_{x}, u_{x} e_{y}=e_{y} u_{x}$, and $u_{y} e_{x}=e_{x} u_{y}$. In view of $G(R)$ being Abelian, $u_{x} u_{y}=u_{y} u_{x}$ and $x y=y x$. Implications remained are obtained by Theorem 1.3 or obvious. 
The condition " $G(R)$ is an Abelian group" is not superfluous by the existence of noncommutative division rings. The condition "clean" is also not superfluous as we see in the ring in Example 1.7 to follow.

Proposition 1.6. Right (resp. left) unit-duo local rings are right (resp. left) duo.

Proof. Let $R$ be a right unit-duo local ring and $I$ be a right ideal of $R$. Take $r \in R$ and $a \in I$. If $r \in G(R)$, then $r a=a u$ for some $u \in G(R)$ since $R$ is right unit-duo, entailing $r a \in I$.

Let $r \in X(R)$. Then since $R$ is local, $1-r$ is a unit of $R$. Thus $(1-r) a=a v$ for some $v \in G(R)$ since $R$ is

The condition "local ring" in Proposition 1.6 is not superfluous as follows.

Example 1.7. We refer to [17, Theorem 1.3.5, Corollary 2.1.14, and Theorem 2.1.15]. Let $\mathbb{Q}\langle x, y\rangle$ be the free algebra with noncommuting indetermiantes $x, y$ over $\mathbb{Q}$. The first Weyl algebra $A_{1}(\mathbb{Q}) \cong \frac{\mathbb{Q}\langle x, y\rangle}{(y x-x y-1)}, R$ say, is a domain whose invertible elements are nonzero rational numbers (hence central), where $(y x-x y-1)$ is the ideal of $\mathbb{Q}\langle x, y\rangle$ generated by $y x-x y-1$. We identify $x$ and $y$ with their images in $R$ for simplicity. $A_{1}(\mathbb{Q})$ is neither left nor right duo. In fact, $R x$ (resp., $x R$ ) is not right (resp., not left) ideal since $x y \notin R x$ (resp., $y x \notin x R$ ). Thus $R$ is neither left nor right duo. But $R$ is unit-duo since $[f]_{\ell}=[f]_{r}=\mathbb{Q}^{*} f$ for any $f \in R$, noting $G(R)=Q^{*}$.

It is well-known that the group ring $R=K G$ is left duo if and only if it is right duo, where $K$ is a commutative ring and $G$ be any group. In fact, $a b=\left((a b)^{*}\right)^{*}=\left(b^{*} a^{*}\right)^{*}=\left(c b^{*}\right)^{*}=b c^{*} \in b R$ for some $c \in R$ (if $R$ is left duo), and $a b=\left((a b)^{*}\right)^{*}=\left(b^{*} a^{*}\right)^{*}=\left(a^{*} d\right)^{*}=d^{*} a \in R a$ for some $d \in R$ (if $R$ is right duo), where $a, b \in R$, and $*$ is the standard involution on $R$ defined by $\left(\sum a_{i} g_{i}\right)^{*}=\sum a_{i} g_{i}^{-1}$ for all $a_{i} \in K, g_{i} \in G$. We also note that $K G$ is left unit-duo if and only if it is right unit-duo by Theorem 1.3(4).

Proposition 1.8. Let $K$ be a field of characteristic zero and $R$ be the group ring $K Q_{8}$, where $Q_{8}$ is the quaternion group. Then the following conditions are equivalent:

(1) $R$ is right (left) duo;

(2) $R$ is right (left) unit-duo;

(3) $R$ is Abelian;

(4) $R$ is IFP.

Proof. By [18, Theorem 7.4.6 and Lemma 7.4.9], $R$ is isomorphic to $K \oplus K \oplus$ $K \oplus K \oplus H(K)$ such that $H(K)$ is either a division ring $D$ or $\operatorname{Mat}_{2}(K)$. (2) $\Rightarrow(3)$ follows from Theorem 1.3(1). If $R$ is Abelian, then $H(K)$ must be a division ring. This entails $R \cong K \oplus K \oplus K \oplus K \oplus D$, so $R$ is both duo and unit-duo. $(1) \Rightarrow(4)$ and $(4) \Rightarrow(3)$ are clear. 
Note that the ring $R$ in Proposition 1.8 is not a domain but a reduced ring as can be seen by $(2 \cdot 1+2 \cdot-1)(1 \cdot 1+(-1) \cdot-1)=0$.

\section{Noncommutative right unit-duo rings of minimal order}

In this section we find basic examples of right unit-duo rings, seeing that the class of noncommutative right unit-duo rings of minimal order is completely determined up to isomorphism.

Due to Lambek [14], a ring $R$ is called symmetric if $r s t=0$ implies $r t s=0$ for all $r, s, t \in R$. Symmetric rings are clearly IFP, but the converse need not hold by [12, Example 1.10]. The class of symmetric rings contains both commutative rings and reduced rings.

Lemma 2.1. (1) [20, Corollary 6] A ring $R$ is a noncommutative local ring of minimal order if and only if $R$ is a noncommutative IFP ring of minimal order.

(2) $[6$, Theorem] Let $R$ be a finite ring of order $m$ with identity. If $m$ has a cube free factorization, then $R$ is a commutative ring.

(3) [6, Proposition] If $R$ is a noncommutative of order $p^{3}, p$ a prime, then $R$ is isomorphic to $U_{2}(G F(p))$.

In [25, Theorem 8], Xu and Xue proved that a noncommutative IFP ring of minimal order is a local ring of order 16, and if $R$ is such a ring, then $R \cong R_{i}$ for some $i \in\{1,2,3,4,5\}$, where $R_{i}$ 's are the rings in the following example.

Example 2.2. In [25, Example 7], we see five kinds of noncommutative finite local rings with 16 elements, with Jacobson radicals of order $\geq 4$. Let $A\langle x, y\rangle$ be the free algebra generated by noncommuting indeterminates $x, y$ over given a commutative ring $A$, and $(x, y)$ denote the ideal of $A\langle x, y\rangle$ generated by $x, y$.

(1) Let $R_{1}=\mathbb{Z}_{2}\langle x, y\rangle / I$, where $I$ is the ideal of $\mathbb{Z}_{2}\langle x, y\rangle$ generated by $x^{3}, y^{3}, y x, x^{2}-x y, y^{2}-x y$. Note $J\left(R_{1}\right)=(x, y)$ and $\left|J\left(R_{1}\right)\right|=8$. Identify $x, y$ with their images in $R_{1}$ for simplicity.

We will show that $R_{1}$ is unit-duo. To see that, we first note that $X\left(R_{1}\right)=J\left(R_{1}\right) \backslash\{0\}=\left\{x, y, x^{2}\left(=y^{2}=x y\right), x+y, x+x^{2}, x^{2}+y, x+y+x^{2}\right\}$, and

$G\left(R_{1}\right)=\left\{1,1+x, 1+y, 1+x^{2}, 1+x+y, 1+x+x^{2}, 1+x^{2}+y, 1+x+y+x^{2}\right\}$.

Then we have

and

$$
\begin{gathered}
{[x]_{\ell}=[x]_{r}=\left[x+x^{2}\right]_{\ell}=\left[x+x^{2}\right]_{r}=\left\{x, x+x^{2}\right\}} \\
{[y]_{\ell}=[y]_{r}=\left[x^{2}+y\right]_{\ell}=\left[x^{2}+y\right]_{r}=\left\{y, y+x^{2}\right\}} \\
{\left[x^{2}\right]_{\ell}=\left\{x^{2}\right\}=\left[x^{2}\right]_{r}}
\end{gathered}
$$

$$
[x+y]_{\ell}=[x+y]_{r}=\left[x+y+x^{2}\right]_{\ell}=\left[x+y+x^{2}\right]_{r}=\left\{x+y, x+y+x^{2}\right\} .
$$

This implies that $R_{1}$ is unit-duo. 
(2) Let $R_{2}=\mathbb{Z}_{4}\langle x, y\rangle / I$, where $I$ is the ideal of $\mathbb{Z}_{4}\langle x, y\rangle$ generated by $x^{3}, y^{3}, y x, x^{2}-x y, x^{2}-2, y^{2}-2,2 x, 2 y$. Identify $x, y$ with their images in $R_{2}$ for simplicity. Note $J\left(R_{2}\right)=(x, y)=2 \mathbb{Z}_{4}+(x, y)$ and $\left|J\left(R_{2}\right)\right|=8$.

Note that $X\left(R_{2}\right)=J\left(R_{2}\right) \backslash\{0\}$,

$$
\begin{gathered}
X\left(R_{2}\right)=\left\{x, y, x^{2}\left(=y^{2}=x y=2\right), x+y, x+x^{2}(=2+x),\right. \\
\left.x^{2}+y(=2+y), x+y+x^{2}(=2+x+y)\right\},
\end{gathered}
$$

and

$$
\begin{gathered}
G\left(R_{2}\right)=\left\{1,1+x, 1+y, 1+x^{2}(=3), 1+x+y, 1+x+x^{2}(=3+x),\right. \\
\left.1+x^{2}+y(=3+y), 1+x+y+x^{2}(=3+x+y)\right\} .
\end{gathered}
$$

So we get the same result as in the computation for $R_{1}$, using the fact that

$$
x=-x, y=-y, x^{2}=-x^{2}=2=y^{2}=-y^{2} \text {, and } 1+J\left(R_{2}\right)=3+J\left(R_{2}\right) .
$$

Thus $R_{2}$ is also unit-duo.

(3) Let $R_{3}=\left\{\left(\begin{array}{cc}a & b \\ 0 & a^{2}\end{array}\right) \in U_{2}\left(G F\left(2^{2}\right)\right) \mid a, b \in G F\left(2^{2}\right)\right\}$. Note

$$
J\left(R_{3}\right)=\left(\begin{array}{cc}
0 & G F\left(2^{2}\right) \\
0 & 0
\end{array}\right) \text { and }\left|J\left(R_{3}\right)\right|=4 .
$$

Then $R_{3}$ is unit-duo since $[a]_{\ell}=[a]_{r}=J\left(R_{3}\right)$ for any $a \in X\left(R_{3}\right)$.

(4) Let $R_{4}=\mathbb{Z}_{2}\langle x, y\rangle / I$, where $I$ is the ideal of $\mathbb{Z}_{2}\langle x, y\rangle$ generated by $x^{3}, y^{2}, y x, x^{2}-x y$. Identify $x, y$ with their images in $R_{4}$ for simplicity. It is simply checked that $R_{4}$ is isomorphic to $D_{3}\left(\mathbb{Z}_{2}\right)$ through the corresponding $x \mapsto E_{12}+E_{23}$ and $y \mapsto E_{23}$. Note that

$$
J\left(R_{4}\right)=(x, y)=\left(\begin{array}{ccc}
0 & \mathbb{Z}_{2} & \mathbb{Z}_{2} \\
0 & 0 & \mathbb{Z}_{2} \\
0 & 0 & 0
\end{array}\right),\left|J\left(R_{4}\right)\right|=8, X\left(R_{4}\right)=J\left(R_{4}\right) \backslash\{0\},
$$

and

$$
G\left(R_{4}\right)=\left(\begin{array}{ccc}
1 & \mathbb{Z}_{2} & \mathbb{Z}_{2} \\
0 & 1 & \mathbb{Z}_{2} \\
0 & 0 & 1
\end{array}\right)=1+J\left(R_{4}\right)
$$

We have $\left[e_{23}\right]_{\ell}=\mathbb{Z}_{2} e_{13}+\mathbb{Z}_{2} e_{23}$ and $\left[e_{23}\right]_{r}=\mathbb{Z}_{2} e_{23} ;$ and $\left[e_{12}\right]_{\ell}=\mathbb{Z}_{2} e_{12}$ and $\left[e_{12}\right]_{r}=\mathbb{Z}_{2} e_{12}+\mathbb{Z}_{2} e_{13}$. Thus $\left[e_{23}\right]_{\ell} \nsubseteq\left[e_{23}\right]_{r}$ and $\left[e_{12}\right]_{r} \nsubseteq\left[e_{12}\right]_{\ell}$, concluding that $R_{4}$ is neither left nor right unit-duo.

(5) Let $R_{5}=\mathbb{Z}_{4}\langle x, y\rangle / I$, where $I$ is the ideal of $\mathbb{Z}_{4}\langle x, y\rangle$ generated by $x^{3}, y^{2}, y x, x^{2}-x y, x^{2}-2,2 x, 2 y$. Identify $x, y$ with their images in $R_{5}$ for simplicity. Note $J\left(R_{5}\right)=(x, y)=2 \mathbb{Z}_{4}+(x, y)$ and $\left|J\left(R_{5}\right)\right|=8$.

Note that $X\left(R_{5}\right)=J\left(R_{5}\right) \backslash\{0\}$,

$$
\begin{array}{r}
X\left(R_{5}\right)=\left\{x, y, x^{2}(=x y=2), x+y, x+x^{2}(=2+x),\right. \\
\left.x^{2}+y(=2+y), x+y+x^{2}(=2+x+y)\right\},
\end{array}
$$

and

$$
G\left(R_{5}\right)=\left\{1,1+x, 1+y, 1+x^{2}(=3), 1+x+y, 1+x+x^{2}(=3+x),\right.
$$




$$
\left.1+x^{2}+y(=3+y), 1+x+y+x^{2}(=3+x+y)\right\} .
$$

We have $[y]_{\ell}=\{y, y+2\}$ and $[y]_{r}=\{y\}$; and $[x+y]_{\ell}=\{x+y\}$ and $[x+y]_{r}=$ $\{x+y, x+y+2\}$, so $R_{5}$ is neither left nor right unit-duo.

We now have a complete structure of noncommutative unit-duo rings of minimal order by help of Example 1.2.

Theorem 2.3. If $R$ is a noncommutative right (left) unit-duo ring of minimal order, then $|R|=16$ and $R$ is isomorphic to $R_{1}, R_{2}$, or $R_{3}$ in Example 1.2.

Proof. Let $R$ be a noncommutative unit-duo ring of minimal order. Then $|R|$ has a cube factorization by Lemma $2.1(2)$, entailing $|R| \geq 2^{3}$. If $|R|=2^{3}$, then $R$ is isomorphic to $U_{2}(G F(2))$ by Lemma 2.1(3). But $U_{2}(G F(2))$ is not unitduo. Consequently we must have $|R| \geq 16$ also by help of Lemma 2.1(2). Thus we must have $|R|=16$ by the existence of the unit-duo ring $R_{1}$ in Example $3.2(3)$.

Since $R$ is semiperfect, $R$ has a finite orthogonal set of local idempotents whose sum is 1 by [13, Proposition 3.7.2], say $R=\sum_{i=1}^{n} e_{i} R$ such that each $e_{i} R e_{i}$ is a local ring. But $R$ is Abelian by Theorem 1.3, and so each $e_{i} R$ is an ideal of $R$ with $e_{i} R=e_{i} R e_{i}$. Since $R$ is a noncommutative unit-duo ring of minimal order, we must have $n=1$ and $R=e_{1} R=e_{1} R e_{1}$, seeing that $R$ is local. This yields that $R$ is isomorphic to an $R_{i}(i=1,2,3,4,5)$ in Example 3.2 by Lemma 2.1(1). But $R_{4}$ and $R_{5}$ are neither left nor right unit-duo, so $R$ is isomorphic to $R_{1}, R_{2}$, or $R_{3}$. The proof of the left case is similar.

Let $R$ be a noncommutative symmetric ring of minimal order. Then $R$ is isomorphic to the unit-duo ring $R_{3}$ in Example 3.2 by [9, Theorem 2.6]. But $R_{1}$ and $R_{2}$ are non-symmetric. Recall that a noncommutative IFP ring of minimal order is a local ring of order 16 and it is isomorphic to some $R_{i}$ in Example 3.2. But $R_{4}$ and $R_{5}$ are not right unit-duo. Also note that the rings $R_{1}, R_{2}$, and $R_{3}$ are unit-duo as well. So one can obtain the following irreversible implications by help of Theorem 2.3 .

Corollary 2.4. (1) A noncommutative symmetric ring of minimal order is a unit-duo ring of minimal order.

(2) A noncommutative right unit-duo ring of minimal order is an IFP ring of minimal order.

(3) A ring is a noncommutative right unit-duo ring of minimal order if and only if it is a noncommutative unit-duo ring of minimal order if and only if it is a noncommutative left unit-duo ring of minimal order.

\section{More properties of right unit-duo rings}

In this section we investigate some properties of right unit-duo rings which are concerned with annihilating conditions. 
Let $R$ be a ring and $x \in R$. We usually write $l_{R}(x)=\{a \in R \mid a x=0\}$ and $r_{R}(x)=\{b \in R \mid x b=0\}$. We use $\operatorname{ann}_{R}(x)$ to denote $l_{R}(x)$ when $l_{R}(x)=r_{R}(x)$, i.e., $\operatorname{ann}_{R}(x)=\{a \in R \mid a x=x a=0\}$.

\section{Lemma 3.1.}

(1) If $[x]_{r}=[y]_{r}$ for $x, y \in R$, then $l_{R}(x)=l_{R}(y)$.

(2) Let $R$ be a right unit-duo ring. If $[x]_{\ell}=[y]_{\ell}$ for $x, y \in R$, then $l_{R}(x)=$ $l_{R}(y)$.

(3) If $[x]_{\ell}=[y]_{\ell}$ for $x, y \in R$, then $r_{R}(x)=r_{R}(y)$.

(4) Let $R$ be a left unit-duo ring. If $[x]_{r}=[y]_{r}$ for $x, y \in R$, then $r_{R}(x)=$ $r_{R}(y)$.

(5) Let $R$ be a unit-duo ring. If $[x]=[y]$, then $\operatorname{ann}_{R}(x)=\operatorname{ann}_{R}(y)$.

Proof. (1) Let $[x]_{r}=[y]_{r}$ for $x, y \in R$. Then $x=y u$ and $y=x v$ for some $u, v \in G(R)$. Letting $a \in l_{R}(x)$ and $b \in l_{R}(y)$, we have $a y=a(x v)=0$ and $b x=b y u=0$; hence $l_{R}(x)=l_{R}(y)$.

(2) Let $[x]_{\ell}=[y]_{\ell}$ for $x, y \in R$. Then since $R$ is right unit-duo, $y \in[x]_{\ell} \subseteq[x]_{r}$ and $x \in[y]_{\ell} \subseteq[y]_{r}$. The remainder of the proof is similar to one of $(1)$.

The proofs of the remainder are analogous to the preceding argument.

The converse of Lemma 3.1(5) need not hold by the following.

Example 3.2. Let $R=D_{2}(\mathbb{Z})=V_{2}(\mathbb{Z})$. Then $R$ is commutative (hence unit-duo). Note that $\left(\begin{array}{ll}a & b \\ 0 & a\end{array}\right)$ is regular if and only if $a \neq 0$ by [7, Lemma 2.1].

Take $x=\left(\begin{array}{ll}0 & 2 \\ 0 & 0\end{array}\right)$ and $y=\left(\begin{array}{ll}0 & 3 \\ 0 & 0\end{array}\right)$ in $R$. Then we have

$$
\operatorname{ann}(x)=\left(\begin{array}{ll}
0 & \mathbb{Z} \\
0 & 0
\end{array}\right)=\operatorname{ann}(y) \text {. }
$$

But $[x] \neq[y]$. In fact,

$$
[x]=\left\{\left(\begin{array}{ll}
0 & 2 \\
0 & 0
\end{array}\right),\left(\begin{array}{cc}
0 & -2 \\
0 & 0
\end{array}\right)\right\},[y]=\left\{\left(\begin{array}{ll}
0 & 3 \\
0 & 0
\end{array}\right),\left(\begin{array}{cc}
0 & -3 \\
0 & 0
\end{array}\right)\right\}
$$

since all units in $R$ are

$$
\left\{\left(\begin{array}{ll}
1 & c \\
0 & 1
\end{array}\right),\left(\begin{array}{cc}
-1 & d \\
0 & -1
\end{array}\right)\right\}
$$

with $c, d \in \mathbb{Z}$.

We see conditions under which the converse of Lemma 3.1(5) holds as follows.

Theorem 3.3. Let $A$ be a principal ideal domain and $R=V_{n}(A)$. Then the following conditions are equivalent:

(1) $[\alpha]=[\beta]$ whenever ann $(\alpha)=\operatorname{ann}_{R}(\beta)$ for $\alpha, \beta \in X(R)$;

(2) $A$ is a field.

Proof. $(1) \Rightarrow(2)$. Assume on the contrary that $A$ is not a field. Then we can take distinct $a, b \in X(A)$ (e.g., distinct nonzero prime elements) such that $a \notin[b]$ and $b \notin[a]$, i.e., $[a] \neq[b]$. 
Note first that $R$ is commutative, and that

$$
\left(\begin{array}{cccccc}
a & b & c & \cdots & e & f \\
0 & a & b & \cdots & g & e \\
\vdots & \vdots & \vdots & \cdots & \vdots & \vdots \\
0 & 0 & 0 & \cdots & a & b \\
0 & 0 & 0 & \cdots & 0 & a
\end{array}\right) \text { is regular if and only if } a \neq 0
$$

by [10, Lemma 2.1].

Take $\alpha=\left(a_{i j}\right)$ and $\beta=\left(b_{i j}\right)$ in $R$ such that

$$
a_{i i}=b_{i i}=0 \text { for all } i \text { and } a_{i j}=a, b_{i j}=b \text { for all } i, j \text { with } i<j .
$$

Then $\alpha, \beta \in X(R)$ and observe

$$
A e_{1 n}=\operatorname{ann}(\alpha)=\operatorname{ann}(\beta)=A e_{1 n}
$$

as well.

Note that $G(R)=\left\{\left(a_{i j}\right) \in V_{n}(A) \mid a_{i i} \in G(A)\right\}$. This yields

$$
[\alpha]=\left\{\left(c_{i j}\right) \in V_{n}(A) \mid c_{i i}=0 \text { and } c_{i j} \in[a] \text { for all } i, j \text { with } i<j\right\}
$$

and

$$
[\beta]=\left\{\left(d_{i j}\right) \in V_{n}(A) \mid d_{i i}=0 \text { and } d_{i j} \in[b] \text { for all } i, j \text { with } i<j\right\} .
$$

This leads us to have $\beta \notin[\alpha]$ and $\alpha \notin[\beta]$. Thus $[\alpha] \neq[\beta]$, a contradiction to the condition (1).

$(2) \Rightarrow(1)$. Let $A$ be a field. It suffices to examine the elements in $X(R)$. Recall that every matrix in $X(R)$ is a nonzero matrix in $V_{n}(A)$ with zero diagonal. Let

$$
\gamma=\left(\begin{array}{cccccc}
0 & a_{1} & a_{2} & \cdots & a_{n-2} & a_{n-1} \\
0 & 0 & a_{1} & \cdots & a_{n-3} & a_{n-2} \\
\vdots & \vdots & \vdots & \cdots & \vdots & \vdots \\
0 & 0 & 0 & \cdots & a_{1} & a_{2} \\
0 & 0 & 0 & \cdots & 0 & a_{1} \\
0 & 0 & 0 & \cdots & 0 & 0
\end{array}\right) \in X(R)
$$

Note $l_{R}(m)=r_{R}(m)=a n n_{R}(m)$ for all $m \in R$.

If $a_{1} \neq 0$, then $\operatorname{ann}_{R}(\gamma)=A e_{1 n}$.

If $a_{1}=0$ and $a_{2} \neq 0$, then $\operatorname{ann}_{R}(\gamma)=A e_{1 n}+A\left(e_{1(n-1)}+e_{2 n}\right)$.

Inductively, if $a_{1}=\cdots=a_{k-1}=0$ and $a_{k} \neq 0$, then

$$
\begin{aligned}
\operatorname{ann}_{R}(\gamma)= & A e_{1 n}+A\left(e_{1(n-1)}+e_{2 n}\right)+\cdots+A\left(e_{1(n-k)}+e_{2(n-k+1)}\right. \\
& \left.+\cdots+e_{(k-1)(n-1)}+e_{k n}\right) .
\end{aligned}
$$

This result leads us to conclude that $s=t$ whenever $a n n_{R}(\alpha)=a n n_{R}(\beta)$ for $\alpha, \beta \in X(R)$, where $\alpha=\left(a_{i j}\right)$ and $\beta=\left(b_{i j}\right)$ such that $a_{1}=\cdots=a_{s-1}=0$, $a_{s} \neq 0$, and $b_{1}=\cdots=b_{t-1}=0, b_{t} \neq 0$. Thus we have $[\alpha]=[\beta]$, using the condition that $A$ is a field. 
We examine the property of a $\operatorname{ring} R$ when $R[x]$ is right unit-duo.

Proposition 3.4. (1) Let $R$ be a ring and suppose that $R[x]$ is right unit-duo. Then $a u=u a$ for $a \in R$ and $u \in G(R)$.

(2) Let $R$ be a division ring and $R[x]$ is right unit-duo. Then $R$ is a field.

Proof. (1) Let $a \in R$ and $u \in G(R)$. Since $R[x]$ is right unit-duo, $u(a+x)=$ $(a+x) u^{\prime}$ for some $u^{\prime} \in G(R[x])$, forcing $u^{\prime} \in G(R)$. This yields $u=u^{\prime}$ and $u a=a u^{\prime}=a u$. (2) is an immediate consequence of (1).

In closing, we pose the following question:

Question. Are right duo rings right unit-duo when they are not domains?

Acknowledgments. The authors thank the referee for very careful reading of the manuscript and many valuable suggestions that improved the paper by much. This work was supported by a 2-Year Research Grant of Pusan National University.

\section{References}

[1] H. E. Bell, Near-rings in which each element is a power of itself, Bull. Austral. Math. Soc. 2 (1970), 363-368.

[2] H. E. Bell and Y. Li, Duo group rings, J. Pure Appl. Algebra 209 (2007), no. 3, 833-838.

[3] H. H. Brungs, Three questions on duo rings, Pacific J. Math. 58 (1975), no. 2, 345-349.

[4] V. Camillo and D. A. Khurana, A characterization of unit regular rings, Comm. Algebra 29 (2001), no. 5, 2293-2295.

[5] V. P. Camillo and H.-P. Yu, Exchange rings, units and idempotents, Comm. Algebra 22 (1994), no. 12, 4737-4749.

[6] K. E. Eldridge, Orders for finite noncommutative rings with unity, Amer. Math. Monthly 73 (1966), 512-514.

[7] E. H. Feller, Properties of primary noncommutative rings, Trans. Amer. Math. Soc. 89 (1958), 79-91.

[8] K. R. Goodearl, Von Neumann Regular Rings, Pitman, London, 1979.

[9] C. Huh, H. K. Kim, N. K. Kim, and Y. Lee, Basic examples and extensions of symmetric rings, J. Pure Appl. Algebra 202 (2005), no. 1-3, 154-167.

[10] S. U. Hwang, N. K. Kim, and Y. Lee, On rings whose right annihilators are bounded, Glasg. Math. J. 51 (2009), no. 3, 539-559.

[11] Y. C. Jeon, H. K. Kim, Y. Lee, and J. S. Yoon, On weak Armendariz rings, Bull. Korean Math. Soc. 46 (2009), no. 1, 135-146.

[12] N. K. Kim and Y. Lee, Extensions of reversible rings, J. Pure Appl. Algebra 185 (2003), no. 1-3, 207-223.

[13] J. Lambek, Lectures on Rings and Modules, Blaisdell Publishing Company, Waltham, 1966.

[14] On the representation of modules by sheaves of factor modules, Canad. Math. Bull. 14 (1971), 359-368.

[15] Y. Lee, On generalizations of commutativity, Comm. Algebra 43 (2015), no. 4, 16871697.

[16] G. Marks, Reversible and symmetric rings, J. Pure Appl. Algebra 174 (2002), no. 3, $311-318$.

[17] J. C. McConnell and J. C. Robson, Noncommutative Noetherian Rings, John Wiley \& Sons Ltd., Chichester, New York, Brisbane, Toronto, Singapore, 1987. 
[18] C. P. Milies and S. K. Sehgal, An Introduction to Group Rings, Kluwer Academic Publishers, Dordrecht, 2002.

[19] L. Motais de Narbonne, Anneaux semi-commutatifs et unis riels anneaux dont les id aux principaux sont idempotents, Proceedings of the 106th National Congress of Learned Societies (Perpignan, 1981), Bib. Nat., Paris (1982), 71-73.

[20] S. B. Nam, Finite local rings of order $\leq 16$ with nonzero Jacobson radical, Korean J. Math. 21 (2013), 23-28.

[21] W. K. Nicholson, Lifting idempotents and exchange rings, Trans. Amer. Math. Soc. 229 (1977), 269-278.

[22] M. B. Rege and S. Chhawchharia, Armendariz rings, Proc. Japan Acad. Ser. A Math. Sci. 73 (1997), no. 1, 14-17.

[23] G. Shin, Prime ideals and sheaf representation of a pseudo symmetric ring, Trans. Amer. Math. Soc. 184 (1973), 43-60.

[24] G. Thierrin, On duo rings, Canad. Math. Bull. 3 (1960), 167-172.

[25] L. Xu and W. Xue, Structure of minimal non-commutative zero-insertive rings, Math. J. Okayama Univ. 40 (1998), 69-76.

JUNCHEOL HAN

Department of Mathematics Education

Pusan National University

Pusan 609-735, Korea

E-mail address: jchan@pusan.ac.kr

YANG LEE

Department of Mathematics Education

Pusan National University

Pusan 609-735, Korea

E-mail address: ylee@pusan.ac.kr

SANGWON PARK

Department of Mathematics

DONG-A UNIVERSITY

Pusan 604-714, KoreA

E-mail address: swpark@donga.ac.kr 